\title{
THE COMPETITION NUMBERS OF HAMMING GRAPHS WITH DIAMETER AT MOST THREE
}

\author{
Boram Park And Yoshio SAno
}

\begin{abstract}
The competition graph of a digraph $D$ is a graph which has the same vertex set as $D$ and has an edge between $x$ and $y$ if and only if there exists a vertex $v$ in $D$ such that $(x, v)$ and $(y, v)$ are $\operatorname{arcs}$ of $D$. For any graph $G, G$ together with sufficiently many isolated vertices is the competition graph of some acyclic digraph. The competition number $k(G)$ of a graph $G$ is defined to be the smallest number of such isolated vertices. In general, it is hard to compute the competition number $k(G)$ for a graph $G$ and it has been one of important research problems in the study of competition graphs. In this paper, we compute the competition numbers of Hamming graphs with diameter at most three.
\end{abstract}

\section{Introduction and main results}

The notion of a competition graph was introduced by Cohen [2] in connection with a problem in ecology (see also [3]). The competition graph $C(D)$ of a digraph $D$ is the (simple undirected) graph which has the same vertex set as $D$ and has an edge between vertices $u$ and $v$ if and only if there is a vertex $x$ in $D$ such that $(u, x)$ and $(v, x)$ are $\operatorname{arcs}$ of $D$. For any graph $G, G$ together with sufficiently many isolated vertices is the competition graph of an acyclic digraph. Roberts [16] defined the competition number $k(G)$ of a graph $G$ to be the smallest number $k$ such that $G$ together with $k$ isolated vertices is the competition graph of an acyclic digraph. Opsut [12] showed that the computation of the competition number of a graph is an NP-hard problem. In the study of competition graphs, it has been one of important research problems to compute the competition numbers for various graph classes (see [1], [4], [5], [6], [7], [9], [11], [16], [18] for graphs whose competition numbers are known). For

Received February 22, 2009; Revised November 4, 2010.

2010 Mathematics Subject Classification. Primary 05C99.

Key words and phrases. competition graph, competition number, edge clique cover, Hamming graph.

This work was supported by the Korea Research Foundation Grant funded by the Korean Government (MOEHRD) (KRF-2008-531-C00004). The first author was supported by Seoul Fellowship. The second author was supported by JSPS Research Fellowships for Young Scientists. 
some special graph families, we have explicit formulae for computing competition numbers. For example, if $G$ is a chordal graph without isolated vertices, then $k(G)=1$, and if $G$ is a nontrivial triangle-free connected graph, then $k(G)=|E(G)|-|V(G)|+2$ (see $[16])$.

Recently, it has been concerned to find the competition numbers of interesting graph families used in many areas of mathematics and computer science (see [8], [10], [13], [14], [15], [17]). Hamming graphs are known as an interesting graph family in connection with error-correcting codes, association schemes, and several branches of mathematics. For a positive integer $q$, we denote the $q$-set $\{1,2, \ldots, q\}$ by $[q]$. Also we denote the set of $n$-tuples over $[q]$ by $[q]^{n}$. For positive integers $n$ and $q$, the Hamming graph $H(n, q)$ is the graph which has the vertex set $[q]^{n}$ and in which two vertices $x=\left(x_{1}, x_{2}, \ldots, x_{n}\right)$ and $y=\left(y_{1}, y_{2}, \ldots, y_{n}\right)$ are adjacent if $d_{H}(x, y)=1$, where $d_{H}:[q]^{n} \times[q]^{n} \rightarrow \mathbb{Z}$ is the Hamming distance defined by

$$
d_{H}(x, y):=\left|\left\{i \in[n] \mid x_{i} \neq y_{i}\right\}\right| .
$$

Note that the diameter of the Hamming graph $H(n, q)$ is equal to $n$ if $q \geq 2$. Since the Hamming graph $H(n, q)$ is an $n(q-1)$-regular graph with $q^{n}$ vertices, it follows that the number of edges of the Hamming graph $H(n, q)$ is equal to $\frac{1}{2} n(q-1) q^{n}$.

In this paper, we study the competition numbers of Hamming graphs. If $q=1$, then $H(n, 1)$ is $K_{1}$ and so the following holds:

Proposition 1. For $n \geq 1$, we have $k(H(n, 1))=0$.

If $q=2$, then since $H(n, 2)$ triangle-free and connected, we have

$$
\begin{aligned}
k(H(n, 2)) & =|E(H(n, 2))|-|V(H(n, 2))|+2 \\
& =n 2^{n-1}-2^{n}+2 \\
& =(n-2) 2^{n-1}+2 .
\end{aligned}
$$

Proposition 2. For $n \geq 1$, we have $k(H(n, 2))=(n-2) 2^{n-1}+2$.

If $n=1$, then $H(1, q)$ is the complete graph $K_{q}$ with $q$ vertices and so the following holds:

Proposition 3. For $q \geq 2$, we have $k(H(1, q))=1$.

However, in general, it is not easy to compute $k(H(n, q))$. In this paper, we give the exact values of $k(H(2, q))$ and $k(H(3, q))$. Our main results are the following:

Theorem 4. For $q \geq 2$, we have $k(H(2, q))=2$.

Theorem 5. For $q \geq 3$, we have $k(H(3, q))=6$.

We use the following notation and terminology in this paper. For a digraph $D$, a sequence $v_{1}, v_{2}, \ldots, v_{n}$ of the vertices of $D$ is called an acyclic ordering of $D$ if $\left(v_{i}, v_{j}\right) \in A(D)$ implies $i>j$. It is well-known that a digraph $D$ is acyclic 
if and only if there exists an acyclic ordering of $D$. For a digraph $D$ and a vertex $v$ of $D$, we define the out-neighborhood $N_{D}^{+}(v)$ of $v$ in $D$ to be the set $\{w \in V(D) \mid(v, w) \in A(D)\}$, and the in-neighborhood $N_{D}^{-}(v)$ of $v$ in $D$ to be the set $\{w \in V(D) \mid(w, v) \in A(D)\}$. A vertex in the out-neighborhood $N_{D}^{+}(v)$ of a vertex $v$ in a digraph $D$ is called a prey of $v$ in $D$. For a graph $G$ and a vertex $v$ of $G$, we define the open neighborhood $N_{G}(v)$ of $v$ in $G$ to be the set $\{u \in V(G) \mid u v \in E(G)\}$, and the closed neighborhood $N_{G}[v]$ of $v$ in $G$ to be the set $N_{G}(v) \cup\{v\}$. We denote the subgraph of $G$ induced by $N_{G}(v)$ (resp. $N_{G}[v]$ ) by the same symbol $N_{G}(v)$ (resp. $N_{G}[v]$ ).

For a clique $S$ of a graph $G$ and an edge $e$ of $G$, we say $e$ is covered by $S$ if both of the endpoints of $e$ are contained in $S$. An edge clique cover of a graph $G$ is a family of cliques of $G$ such that each edge of $G$ is covered by some clique in the family. The edge clique cover number $\theta_{E}(G)$ of a graph $G$ is the minimum size of an edge clique cover of $G$. An edge clique cover of $G$ is called a minimum edge clique cover of $G$ if its size is equal to $\theta_{E}(G)$. A vertex clique cover of a graph $G$ is a family of cliques of $G$ such that each vertex of $G$ is contained in some clique in the family. The smallest size of a vertex clique cover of $G$ is called the vertex clique cover number, and is denoted by $\theta_{V}(G)$.

We denote a path with $n$ vertices by $P_{n}$, a cycle with $n$ vertices by $C_{n}$, a graph with $n$ vertices and no edges by $I_{n}$, and a complete multipartite graph by $K_{n_{1}, \ldots, n_{m}}$.

\section{Proofs of Theorems 4 and 5}

\subsection{Cliques in a Hamming graph}

Let $\pi_{j}:[q]^{n} \rightarrow[q]^{n-1}$ be a map defined by

$$
\left(x_{1}, \ldots, x_{j-1}, x_{j}, x_{j+1}, \ldots, x_{n}\right) \mapsto\left(x_{1}, \ldots, x_{j-1}, x_{j+1}, \ldots, x_{n}\right) .
$$

For $j \in[n]$ and $p \in[q]^{n-1}$, let

$$
S_{j}(p):=\pi_{j}^{-1}(p)=\left\{x \in[q]^{n} \mid \pi_{j}(x)=p\right\} .
$$

Then $S_{j}(p)$ is a clique of $H(n, q)$ with size $q$. Let

$$
\mathcal{F}(n, q):=\left\{S_{j}(p) \mid j \in[n], p \in[q]^{n-1}\right\} .
$$

Then $\mathcal{F}(n, q)$ is the family of maximal cliques of $H(n, q)$.

Lemma 6. Let $n \geq 2$ and $q \geq 2$, and let $K$ be a clique of $H(n, q)$ with size at least 2. Then there exists a unique maximal clique $S$ of $H(n, q)$ containing $K$.

Proof. Since $\mathcal{F}(n, q)$ is the family of maximal cliques of $H(n, q)$, it is sufficient to show that there is a unique maximal clique in $\mathcal{F}(n, q)$ containing $K$.

Take a vertex $x=\left(x_{1}, x_{2}, \ldots, x_{n}\right) \in K$. Now we will show that there exists a unique integer $j$ such that $\pi_{j}(x)=\pi_{j}(y)$ for all vertices $y \in K \backslash\{x\}$. Take a vertex $y=\left(y_{1}, y_{2}, \ldots, y_{n}\right) \in K \backslash\{x\}$. Since $K$ is a clique, $x$ and $y$ are adjacent. Then there is a unique integer $j \in[n]$ such that $x_{j} \neq y_{j}$ and $\pi_{j}(x)=\pi_{j}(y)$. Suppose that there is a vertex $z=\left(z_{1}, z_{2}, \ldots, z_{n}\right) \in K \backslash\{x, y\}$ such that 
$\pi_{j}(z) \neq \pi_{j}(x)$. Since $x$ and $z$ are adjacent, there is $j_{1} \in[n]$ with $j_{1} \neq j$ such that $\pi_{j_{1}}(x)=\pi_{j_{1}}(z)$, and thus $x_{j}=z_{j}$. Since $y$ and $z$ are adjacent, there is $j_{2} \in[n]$ with $j_{2} \neq j$ such that $\pi_{j_{2}}(y)=\pi_{j_{2}}(z)$, and thus $y_{j}=z_{j}$. Thus we have $x_{j}=z_{j}=y_{j}$, which contradicts to the fact that $x_{j} \neq y_{j}$. Therefore, $\pi_{j}(z)=\pi_{j}(x)$.

It implies that $j$ is the unique integer such that $\pi_{j}(x)=\pi_{j}(y)$ for all $y \in K$. Hence $K$ is contained in $S_{j}\left(\pi_{j}(x)\right) \in \mathcal{F}(n, q)$. From the uniqueness of $j \in[n]$ and the fact that $\pi_{j}(x)$ does not depend on the choice of $x \in K$, it follows that $S_{j}\left(\pi_{j}(x)\right)$ is the unique maximal clique containing $K$.

Lemma 7. The following hold:

(a) The family $\mathcal{F}(n, q)$ defined by (2) is an edge clique cover of $H(n, q)$.

(b) The edge clique cover number of $H(n, q)$ is equal to $n q^{n-1}$.

(c) Any minimum edge clique cover of $H(n, q)$ consists of edge disjoint maximum cliques.

Proof. Since each edge is contained in a maximal clique and $\mathcal{F}(n . q)$ is the family of maximal cliques in $H(n, q)$, it follows that $\mathcal{F}(n, q)$ is an edge clique cover of $H(n, q)$.

Let $\mathcal{E}$ be a minimum edge clique cover of $H(n, q)$, that is, $\theta_{E}(H(n, q))=|\mathcal{E}|$. Since $\mathcal{F}(n, q)$ is an edge clique cover with $|\mathcal{F}(n, q)|=n q^{n-1}$, we have $|\mathcal{E}| \leq$ $n q^{n-1}$. Now we will show that $|\mathcal{E}| \geq n q^{n-1}$. For a clique $S$, let $E(S):=\left(\begin{array}{l}S \\ 2\end{array}\right)$. Since $\mathcal{E}$ is an edge clique cover of $H(n, q)$, it holds that

$$
|E(H(n, q))| \leq \sum_{S \in \mathcal{E}}|E(S)|,
$$

and the equality holds if and only if none of two distinct cliques in $\mathcal{E}$ have a common edge. Since the maximum size of a clique of $H(n, q)$ is equal to $q$, we have $|E(S)| \leq\left(\begin{array}{l}q \\ 2\end{array}\right)$ for each $S \in \mathcal{E}$. Therefore,

$$
\sum_{S \in \mathcal{E}}|E(S)| \leq\left(\begin{array}{l}
q \\
2
\end{array}\right) \times|\mathcal{E}|,
$$

and the equality holds if and only if any element of $\mathcal{E}$ is a maximum clique in $H(n, q)$. Since $|E(H(n, q))|=\frac{1}{2} n(q-1) q^{n}=\left(\begin{array}{l}q \\ 2\end{array}\right) \times n q^{n-1}$, it follows from (3) and (4) that $n q^{n-1} \leq|\mathcal{E}|$, or $n q^{n-1}=|\mathcal{E}|$.

Moreover, since two equalities of (3) and (4) hold, we can conclude that any minimum edge clique cover of $H(n, q)$ consists of edge disjoint maximum cliques.

Corollary 8. The family $\mathcal{F}(n, q)$ defined by $(2)$ is a minimum edge clique cover of $H(n, q)$.

Proof. It follows from the fact that $|\mathcal{F}(n, q)|=n q^{n-1}$ and Lemma 7 . 


\subsection{Proof of Theorem 4}

In this subsection, we give a proof of Theorem 4 .

Lemma 9. Let $n \geq 2$ and $q \geq 2$. For any vertex $x$ of $H(n, q)$, we have $\theta_{V}\left(N_{H(n, q)}(x)\right)=n$.

Proof. Take any vertex $x \in[q]^{n}$ of $H(n, q)$. Then the vertex $x$ is adjacent to a vertex $y$ such that $\pi_{j}(x)=\pi_{j}(y)$ for some $j \in[n]$. We can easily check from the definition of $H(n, q)$ that, for any $j \in[n]$, the set $S_{j}\left(\pi_{j}(x)\right):=\left\{y \in[q]^{n} \mid\right.$ $\left.\pi_{j}(x)=\pi_{j}(y)\right\}$ forms a clique of $H(n, q)$. Since $N_{H(n, q)}(x)=\cup_{j \in[n]} S_{j}\left(\pi_{j}(x)\right) \backslash$ $\{x\}$, the family $\left\{S_{j}\left(\pi_{j}(x)\right) \mid j \in[n]\right\}$ is a vertex clique cover of $N_{H(n, q)}(x)$ and so $\theta_{V}\left(N_{H(n, q)}(x)\right) \leq n$.

Moreover, note that $S_{j}\left(\pi_{j}(x)\right) \cap S_{j^{\prime}}\left(\pi_{j^{\prime}}(x)\right)=\{x\}$ for $j, j^{\prime} \in[n]$ where $j \neq j^{\prime}$. Take $y_{j} \in S_{j}\left(\pi_{j}(x)\right) \backslash\{x\}$ for each $j \in[n]$. Then $y_{1}, y_{2}, \ldots, y_{n}$ are $n$ vertices of $N_{H(n, q)}(x)$ such that no two of them can be covered by a same clique and so $\theta_{V}\left(N_{H(n, q)}(x)\right) \geq n$.

Opsut showed the following lower bound for the competition number of a graph.

Theorem 10 ([12]). For a graph $G$, it holds that $k(G) \geq \min \left\{\theta_{V}\left(N_{G}(v)\right)\right.$ $v \in V(G)\}$.

Corollary 11. If $n \geq 2$ and $q \geq 2$, then $k(H(n, q)) \geq n$.

Proof. It immediately follows from Lemma 9 and Theorem 10.

We define a total order $\prec$ on the set $[q]^{n}$ as follows. Take two distinct elements $x=\left(x_{1}, x_{2}, \ldots, x_{n}\right)$ and $y=\left(y_{1}, y_{2}, \ldots, y_{n}\right)$ in $[q]^{n}$. Then we define $x \prec y$ if there exists $j \in[n]$ such that $x_{i}=y_{i}$ for $i \leq j-1$ and $x_{j}<y_{j}$. The lexicographic ordering of $[q]^{n}$ is the ordering $v_{1}, v_{2}, \ldots, v_{q^{n}}$ such that $v_{1} \prec v_{2} \prec$ $\cdots \prec v_{q^{n}}$.

Proof of Theorem 4. By Corollary 11, it follows that $k(H(2, q)) \geq 2$. Now we show that $k(H(2, q)) \leq 2$. We define a digraph $D$ as follows:

$$
\begin{aligned}
V(D)= & V(H(2, q)) \cup\left\{z_{1}, z_{2}\right\}, \\
A(D)= & \left(\bigcup_{i=2}^{q}\left\{(x,(1, i-1)) \mid x \in S_{1}(i)\right\}\right) \cup\left(\bigcup_{i=2}^{q}\left\{(x,(i-1, q)) \mid x \in S_{2}(i)\right\}\right) \\
& \cup\left\{\left(x, z_{1}\right) \mid x \in S_{1}(1)\right\} \cup\left\{\left(x, z_{2}\right) \mid x \in S_{2}(1)\right\},
\end{aligned}
$$

where $S_{j}(i)$ with $j \in\{1,2\}$ and $i \in[q]$ is the clique of $H(2, q)$ defined by (1). Since $\left\{N_{D}^{-}(v)|v \in V(D),| N_{D}^{-}(v) \mid \geq 2\right\}=\mathcal{F}(2, q)$, it is easy to check that $C(D)=H(2, q) \cup\left\{z_{1}, z_{2}\right\}$. In addition, the ordering obtained by adding $z_{1}, z_{2}$ on the head of the lexicographic ordering of $V(H(2, q))$ is an acyclic ordering of $D$. To see why, take an $\operatorname{arc}(x, y) \in A(D)$. If $x \in S_{1}(1)$ or $x \in S_{2}(1)$, then $y$ is either $z_{1}$ or $z_{2}$. If $x \in S_{1}(i)$ or $x \in S_{2}(i)$ for some $2 \leq i \in[q]$, then $x=(l, i)$ 
or $x=(i, l)$ for some $l \in[q]$. Since $y=(1, i-1)$ or $y=(i-1, q)$, we have $y \prec x$. Therefore $D$ is acyclic. Hence we have $k(H(2, q)) \leq 2$.

\subsection{Proof of Theorem 5}

In this subsection, we give a proof of Theorem 5 .

\subsection{1. $k(H(3, q)) \geq 6(q \geq 3)$}

First, we improve the lower bound for the competition number of a Hamming graph $H(n, q)$ given in Corollary 11 in the case where $n \geq 3$ and $q \geq 3$.

Lemma 12. Let $n \geq 2$ and $q \geq 2$. Let $D$ be an acyclic digraph such that $C(D)=H(n, q) \cup I_{k}$ with $I_{k}=\left\{z_{1}, z_{2}, \ldots, z_{k}\right\}$. Let $z_{1}, z_{2}, \ldots, z_{k}, v_{1}, v_{2}, \ldots, v_{q^{n}}$ be an acyclic ordering of $D$. Let $U_{i}:=\left\{v_{1}, \ldots, v_{i}\right\}$ for $i \in\left\{1, \ldots, q^{n}\right\}$. Then

$$
\left|\left\{S \in \mathcal{F}(n, q) \mid S \cap U_{i} \neq \emptyset\right\}\right| \leq k+i-1 .
$$

Proof. Let

$$
\begin{aligned}
\mathcal{N}(D) & :=\left\{N_{D}^{-}(x)|x \in V(D),| N_{D}^{-}(x) \mid \geq 2\right\} \\
\mathcal{S}_{i} & :=\left\{N_{D}^{-}(x)\left|x \in U_{i-1} \cup I_{k},\right| N_{D}^{-}(x) \mid \geq 2\right\}, \\
\mathcal{K}_{i} & :=\left\{K \in \mathcal{N}(D) \mid K \cap U_{i} \neq \emptyset\right\} .
\end{aligned}
$$

Since $D$ is acyclic, it holds that $\mathcal{K}_{i}=\left\{K \in \mathcal{S}_{i} \mid K \cap U_{i} \neq \emptyset\right\}$. Since $\left|\mathcal{S}_{i}\right| \leq$ $k+i-1$, it follows that

$$
\left|\mathcal{K}_{i}\right|=\left|\left\{K \in \mathcal{S}_{i} \mid K \cap U_{i} \neq \emptyset\right\}\right| \leq\left|\mathcal{S}_{i}\right| \leq k+i-1 .
$$

For each $K \in \mathcal{K}_{i}$, there exists a unique element in $\mathcal{F}(n, q)$ containing $K$ by Lemma 6 , we denote it by $S_{K}$. From (5), it remains to show that

$$
\left|\left\{S \in \mathcal{F}(n, q) \mid S \cap U_{i} \neq \emptyset\right\}\right| \leq\left|\mathcal{K}_{i}\right| .
$$

Take $S \in \mathcal{F}(n, q)$ such that $S \cap U_{i} \neq \emptyset$. Then there exists a vertex $x$ in $S \cap U_{i}$. Since $q \geq 2$, there exists a vertex $y \in S \backslash\{x\}$. Since $C(D)=H(n, q) \cup I_{k}$ and the vertices $x$ and $y$ are adjacent, there is a common prey $u$ of $x$ and $y$ in $D$. Then $x \in N_{D}^{-}(u) \cap U_{i}$ and so $N_{D}^{-}(u) \in \mathcal{K}_{i}$. Since $N_{D}^{-}(u)$ contains $x$ and $y$, $S_{N_{D}^{-}(u)}$ is a maximal clique containing $x$ and $y$. Then both $S$ and $S_{N_{D}^{-}(u)}$ are maximal cliques containing $x$ and $y$. By Lemma 6 , we have $S=S_{N_{D}^{-}(u)}$, which implies that $S \in\left\{S_{K} \mid K \in \mathcal{K}_{i}\right\}$. It follows that

$$
\left\{S \in \mathcal{F}(n, q) \mid S \cap U_{i} \neq \emptyset\right\} \subseteq\left\{S_{K} \mid K \in \mathcal{K}_{i}\right\},
$$

and together with $\left|\left\{S_{K} \mid K \in \mathcal{K}_{i}\right\}\right| \leq\left|\mathcal{K}_{i}\right|$, (6) holds. Hence, the lemma holds.

Lemma 13. For $n \geq 3$ and $q \geq 3$, we have $k(H(n, q)) \geq 3 n-4$.

Proof. Let $k$ be the competition number of $H(n, q)$ and let $D$ be an acyclic digraph such that $C(D)=H(n, q) \cup I_{k}$ with $I_{k}=\left\{z_{1}, z_{2}, \ldots, z_{k}\right\}$. Let $z_{1}, z_{2}$, 
$\ldots, z_{k}, v_{1}, v_{2}, \ldots, v_{q^{n}}$ be an acyclic ordering of $D$. Let $U_{3}:=\left\{v_{1}, v_{2}, v_{3}\right\}$. By Lemma 12 , it holds that

$$
\left|\left\{S \in \mathcal{F}(n, q) \mid S \cap U_{3} \neq \emptyset\right\}\right| \leq k+2 .
$$

In addition, it holds that $\left|\left\{S \in \mathcal{F}(n, q) \mid S \cap U_{3} \neq \emptyset\right\}\right| \geq 3 n-2$ whose proof will be shown in next paragraph. Therefore, we have $3 n-2 \leq k+2$, or $k \geq 3 n-4$.

Now it remains to show that $\left|\left\{S \in \mathcal{F}(n, q) \mid S \cap U_{3} \neq \bar{\emptyset}\right\}\right| \geq 3 n-2$. Consider the subgraph of $H(n, q)$ induced by $U_{3}$, say $H$. Then $H$ is isomorphic to one of the following:
(i) $K_{3}$
(ii) $P_{3}$
(iii) $P_{2} \cup I_{1}$
(iv) $I_{3}$.

Case (i) $H \cong K_{3}$ : By Lemma $6, U_{3}$ is contained in exactly one maximal clique. Without loss of generality, we may assume that $U_{3}$ is contained in $S_{1}((\underbrace{1, \ldots, 1}_{n-1}))$, and so we may also assume that

$$
U_{3}=\{(\underbrace{1,1, \ldots, 1}_{n}),(2, \underbrace{1, \ldots, 1}_{n-1}),(3, \underbrace{1, \ldots, 1}_{n-1})\} .
$$

Then the family $\left\{S \in \mathcal{F}(n, q) \mid S \cap U_{3} \neq \emptyset\right\}$ consists of the following $3 n-2$ elements:

$$
S_{1}((\underbrace{1, \ldots, 1}_{n-1})), \quad S_{j}((i, \underbrace{1, \ldots, 1}_{n-2}))(i \in\{1,2,3\}, j \in[n] \backslash\{1\}) .
$$

Case (ii) $H \cong P_{3}$ : Without loss of generality, we may assume that

$$
U_{3}=\{(\underbrace{1, \ldots, 1}_{n}),(2, \underbrace{1, \ldots, 1}_{n-1}),(1,2, \underbrace{1, \ldots, 1}_{n-2})\} .
$$

Then the family $\left\{S \in \mathcal{F}(n, q) \mid S \cap U_{3} \neq \emptyset\right\}$ consists of the following $3 n-2$ elements:

$$
\begin{aligned}
& S_{j}((\underbrace{1, \ldots, 1}_{n-1}))(j \in[n]), \quad S_{j}((2, \underbrace{1, \ldots, 1}_{n-2}))(j \in[n] \backslash\{1\}), \\
& S_{j}((1,2, \underbrace{1, \ldots, 1}_{n-3}))(j \in[n] \backslash\{2\}) .
\end{aligned}
$$

Case (iii) $\boldsymbol{H} \cong \boldsymbol{P}_{\mathbf{2}} \cup \boldsymbol{I}_{\mathbf{1}}$ or (iv) $\boldsymbol{H} \cong \boldsymbol{I}_{\mathbf{3}}$ : Let $v$ be an isolated vertex of $\boldsymbol{H}$. Since the $n$ cliques in $\mathcal{F}(n, q)$ containing $v$ do not contain the other vertices of $U_{3}$, it is sufficient to show that $\left\{S \in \mathcal{F}(n, q) \mid S \cap\left(U_{3} \backslash\{v\}\right) \neq \emptyset\right\}$ has at least $2 n-2$ elements. Since, for each vertex $u \in U_{3} \backslash\{v\}$, there are $n$ cliques in $\mathcal{F}(n, q)$ containing $u$ and there is at most one clique in $\mathcal{F}(n, q)$ containing the two vertices of $U_{3} \backslash\{v\}$. Thus we can conclude that $\left\{S \in \mathcal{F}(n, q) \mid S \cap\left(U_{3} \backslash\{v\}\right) \neq \emptyset\right\}$ has at least $2 n-1$ elements.

We complete the proof.

If $n=3$, then the above lower bound gives $k(H(3, q)) \geq 5$. In this case, however, we can improve the bound as follows. 
Lemma 14. For $q \geq 3$, we have $k(H(3, q)) \geq 6$.

Proof. By Lemma 13, we have $k(H(3, q)) \geq 5$. Suppose that $k(H(3, q))=5$. Then there exists an acyclic digraph $D$ such that $C(D)=H(3, q) \cup I_{5}$ with $I_{5}=\left\{z_{1}, z_{2}, \ldots, z_{5}\right\}$. Let $z_{1}, z_{2}, \ldots, z_{5}, v_{1}, v_{2}, \ldots, v_{q^{3}}$ be an acyclic ordering of $D$. Let $U_{4}:=\left\{v_{1}, v_{2}, v_{3}, v_{4}\right\}$. For convenience, let

$$
\mathcal{A}_{1}:=\left\{S \in \mathcal{F}(3, q) \mid S \cap U_{4} \neq \emptyset\right\}, \quad \mathcal{A}_{2}:=\left\{S \in \mathcal{F}(3, q) \mid v_{5} \in S\right\} .
$$

Now we consider the subgraph $G$ of $H(3, q)$ induced by $U_{4}$. Any graph on 4 vertices is isomorphic to one of the following graphs:
(i) $K_{4}$
(ii) $K_{1,1,2}$
(iii) $K_{4}-E\left(P_{3}\right) \quad$ (iv) $C_{4}$
(v) $P_{4}$
(vi) $K_{1,3}$
(ix) $P_{3} \cup I_{1}$
(x) $K_{2} \cup I_{2}$
(vii) $K_{3} \cup I_{1}$
(viii) $K_{2} \cup K_{2}$

Since $H(3, q)$ does not contain an induced subgraph isomorphic to $K_{1,1,2}$ by Lemma $6, G$ is one of the above graphs except (ii). For each cases, the number $\left|\mathcal{A}_{1}\right|$ is given as follows:
(i) 9
(ii) -
(iii) $9 \quad$ (iv) 8
(v) 9
(vi) 9
(vii) 10 (viii) 10
(ix) 10
(x) 11
(xi) 12 .

By Lemma 12 , we have $\left|\mathcal{A}_{1}\right| \leq 8$. Therefore $G \cong C_{4}$ and so $\left|\mathcal{A}_{1}\right|=8$. Since each vertex of $H(3, q)$ is contained in exactly 3 cliques in $\mathcal{F}(3, q),\left|\mathcal{A}_{2}\right|=3$. From the fact that

$$
\mathcal{A}_{1} \cup \mathcal{A}_{2}=\left\{S \in \mathcal{F}(3, q) \mid S \cap\left(U_{4} \cup\left\{v_{5}\right\}\right) \neq \emptyset\right\},
$$

it holds that $\left|\mathcal{A}_{1} \cup \mathcal{A}_{2}\right| \leq 9$ by Lemma 12 . Since $\left|\mathcal{A}_{1}\right|=8,\left|\mathcal{A}_{2}\right|=3$, and $\left|\mathcal{A}_{1} \cup \mathcal{A}_{2}\right| \leq 9$, we have $\left|\mathcal{A}_{1} \cap \mathcal{A}_{2}\right|=\left|\mathcal{A}_{1}\right|+\left|\mathcal{A}_{2}\right|-\left|\mathcal{A}_{1} \cup \mathcal{A}_{2}\right| \geq 8+3-9=2$.

Take two distinct cliques $S, S^{\prime} \in \mathcal{A}_{1} \cap \mathcal{A}_{2}$. Then $S \cap U_{4} \neq \emptyset, S^{\prime} \cap U_{4} \neq \emptyset$ and so take $x \in S \cap U_{4}$ and $y \in S^{\prime} \cap U_{4}$. If $x=y$ or $x$ and $y$ are adjacent, then $S=S^{\prime}$ by Lemma 6 . Therefore $x$ and $y$ are not adjacent. Since $G \cong C_{4}$, without loss of generality, we may assume that

$$
U_{4}=\{(1,1,1),(1,1,2),(1,2,2),(1,2,1)\}, \quad x=(1,1,1), \quad y=(1,2,2) .
$$

Since $x$ and $v_{5}$ are adjacent, one of the following holds:

$$
\begin{aligned}
& \pi_{1}(x)=(1,1)=\pi_{1}\left(v_{5}\right), \quad \pi_{2}(x)=(1,1)=\pi_{2}\left(v_{5}\right), \\
& \pi_{3}(x)=(1,1)=\pi_{3}\left(v_{5}\right) .
\end{aligned}
$$

Since $y$ and $v_{5}$ are adjacent, one of the following holds:

$$
\begin{aligned}
& \pi_{1}(y)=(2,2)=\pi_{1}\left(v_{5}\right), \quad \pi_{2}(y)=(1,2)=\pi_{2}\left(v_{5}\right), \\
& \pi_{3}(y)=(1,2)=\pi_{3}\left(v_{5}\right) .
\end{aligned}
$$

However, it is impossible that $v_{5}$ satisfies both one of (8) and one of (9) since $v_{5} \notin U_{4}$. We reach a contradiction. Hence we conclude $k(H(3, q)) \geq 6$. 


\subsection{2. $k(H(3, q)) \leq 6(q \geq 3)$}

Next, we show the upper bound $k(H(3, q)) \leq 6$ for $q \geq 3$. To show the upper bound, we introduce a graph $K_{q_{1}} \square K_{q_{2}} \square K_{q_{3}}$ as an extension of $H(3, q)$.

For graphs $G$ and $H$, the Cartesian product $G \square H$ of $G$ and $H$ is the graph which has the vertex set $V(G) \times V(H)$ and has an edge between two vertices $(g, h)$ and $\left(g^{\prime}, h^{\prime}\right)$ if and only if $g g^{\prime} \in E(G)$ and $h=h^{\prime}$, or $g=g^{\prime}$ and $h h^{\prime} \in$ $E(H)$. Note that the Cartesian product of $n$ complete graphs $K_{q}$ of size $q$ is the Hamming graph $H(n, q)$.

Define

Let $q_{1}, q_{2}, q_{3} \geq 2$ be integers and we consider the graph $K_{q_{1}} \square K_{q_{2}} \square K_{q_{3}}$.

$$
\begin{array}{llrl}
\pi_{1}:\left[q_{1}\right] \times\left[q_{2}\right] \times\left[q_{3}\right] \rightarrow\left[q_{2}\right] \times\left[q_{3}\right], & \left(x_{1}, x_{2}, x_{3}\right) & \mapsto\left(x_{2}, x_{3}\right), \\
\pi_{2}:\left[q_{1}\right] \times\left[q_{2}\right] \times\left[q_{3}\right] \rightarrow\left[q_{1}\right] \times\left[q_{3}\right], & \left(x_{1}, x_{2}, x_{3}\right) & \mapsto\left(x_{1}, x_{3}\right), \\
\pi_{3}:\left[q_{1}\right] \times\left[q_{2}\right] \times\left[q_{3}\right] \rightarrow\left[q_{1}\right] \times\left[q_{2}\right], & \left(x_{1}, x_{2}, x_{3}\right) & \mapsto\left(x_{1}, x_{2}\right) .
\end{array}
$$

For $p_{1} \in\left[q_{1}\right], p_{2} \in\left[q_{2}\right]$, and $p_{3} \in\left[q_{3}\right]$, let

$$
\begin{aligned}
S_{1}\left(\left(p_{2}, p_{3}\right)\right) & :=\left\{x \in\left[q_{1}\right] \times\left[q_{2}\right] \times\left[q_{3}\right] \mid \pi_{1}(x)=\left(p_{2}, p_{3}\right)\right\}, \\
S_{2}\left(\left(p_{1}, p_{3}\right)\right) & :=\left\{x \in\left[q_{1}\right] \times\left[q_{2}\right] \times\left[q_{3}\right] \mid \pi_{2}(x)=\left(p_{1}, p_{3}\right)\right\}, \\
S_{3}\left(\left(p_{1}, p_{2}\right)\right) & :=\left\{x \in\left[q_{1}\right] \times\left[q_{2}\right] \times\left[q_{3}\right] \mid \pi_{3}(x)=\left(p_{1}, p_{2}\right)\right\} .
\end{aligned}
$$

Note that $S_{1}\left(\left(p_{2}, p_{3}\right)\right), S_{2}\left(\left(p_{1}, p_{3}\right)\right)$, and $S_{3}\left(\left(p_{1}, p_{2}\right)\right)$ are maximal cliques of $K_{q_{1}} \square K_{q_{2}} \square K_{q_{3}}$. We denote the set of all maximal cliques $S_{1}\left(\left(p_{2}, p_{3}\right)\right), S_{2}\left(\left(p_{1}, p_{3}\right)\right)$ and $S_{3}\left(\left(p_{1}, p_{2}\right)\right)$ by $\mathcal{F}_{\left(q_{1}, q_{2}, q_{3}\right)}$. Then $\mathcal{F}_{\left(q_{1}, q_{2}, q_{3}\right)}$ is an edge clique cover of $K_{q_{1}} \square K_{q_{2}} \square K_{q_{3}}$.

Lemma 15. For $q_{1}, q_{2}, q_{3} \geq 2$, there exists an acyclic digraph $D$ such that $C(D)=\left(K_{q_{1}} \square K_{q_{2}} \square K_{q_{3}}\right) \cup I_{6}$ and

$$
\left\{N_{D}^{-}(v)|v \in V(D),| N_{D}^{-}(v) \mid \geq 2\right\}=\mathcal{F}_{\left(q_{1}, q_{2}, q_{3}\right)} .
$$

Consequently, we have $k\left(K_{q_{1}} \square K_{q_{2}} \square K_{q_{3}}\right) \leq 6$.

Proof. For any digraph $D$, we define $\mathcal{N}(D):=\left\{N_{D}^{-}(v)|v \in V(D),| N_{D}^{-}(v) \mid \geq\right.$ $2\}$. We prove the lemma by induction on $m=q_{1}+q_{2}+q_{3}$. Since $q_{1}, q_{2}, q_{3} \geq$ 2 , we have $m \geq 6$. Suppose $m=6$, i.e., $q_{1}=q_{2}=q_{3}=2$. Note that $K_{2} \square K_{2} \square K_{2}=H(3,2)$. Since $H(3,2)$ is a triangle-free graph, there exists an acyclic digraph $D$ such that $C(D)=H(3,2) \cup I_{6}$ and that $N_{D}^{-}(v)$ is either the empty set or a maximum clique in $H(3,2)$ for each vertex $v \in V(D)$ (see Figure 1 for an illustration of such a digraph). Thus the statement is true for $m=6$.

Suppose that the statement is true for $m=q_{1}+q_{2}+q_{3}$ where $m \geq 6$. Consider a graph $K_{q_{1}} \square K_{q_{2}} \square K_{q_{3}}$ such that $m+1=q_{1}+q_{2}+q_{3}$. Since $q_{1}+q_{2}+$ $q_{3}>6$, at least one of $q_{1}, q_{2}$, or $q_{3}$ is greater than 2 . Without loss of generality, we may assume that $q_{1}>2$. Now we consider the graph $K_{q_{1}-1} \square K_{q_{2}} \square K_{q_{3}}$ which is a subgraph of $K_{q_{1}} \square K_{q_{2}} \square K_{q_{3}}$. Then by the induction hypothesis, there exists an acyclic digraph $D_{0}$ such that $C\left(D_{0}\right)=\left(K_{q_{1}-1} \square K_{q_{2}} \square K_{q_{3}}\right) \cup I_{6}$ 
$(1,1,2)$

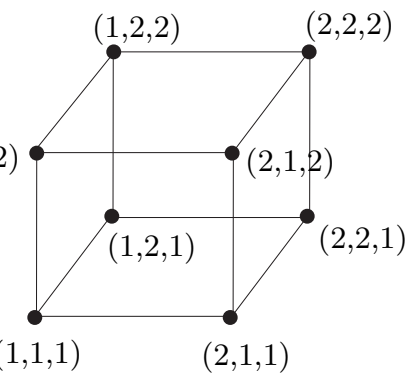

$H(3,2)$

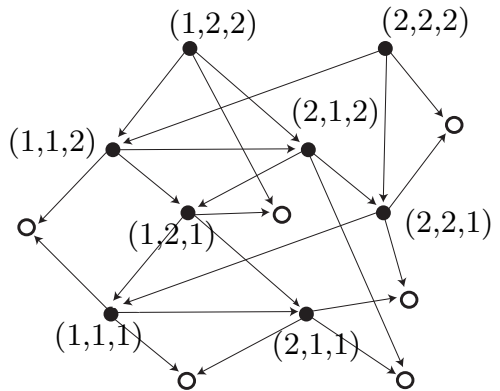

$D$

Figure 1. The Hamming graph $H(3,2)$ and an acyclic digraph $D$ satisfying $C(D)=H(3,2) \cup I_{6}$

and

$$
\mathcal{N}\left(D_{0}\right)=\mathcal{F}_{\left(q_{1}-1, q_{2}, q_{3}\right)} .
$$

Let $v_{1}, v_{2}, \ldots, v_{q_{1} q_{2} q_{3}-q_{2} q_{3}+6}$ be an acyclic ordering of $D_{0}$. For convenience, let $w_{1}:=v_{q_{1} q_{2} q_{3}-q_{2} q_{3}+5}$ and $w_{2}:=v_{q_{1} q_{2} q_{3}-q_{2} q_{3}+6}$.

Let $H^{*}$ be the subgraph of $K_{q_{1}} \square K_{q_{2}} \square K_{q_{3}}$ induced by

$$
\begin{aligned}
V^{*} & :=V\left(K_{q_{1}} \square K_{q_{2}} \square K_{q_{3}}\right)-V\left(K_{q_{1}-1} \square K_{q_{2}} \square K_{q_{3}}\right) \\
& =\left\{\left(q_{1}, p_{2}, p_{3}\right) \mid p_{2} \in\left[q_{2}\right], p_{3} \in\left[q_{3}\right]\right\} .
\end{aligned}
$$

Now we define a digraph $D_{1}$ as follows:

$$
\begin{aligned}
V\left(D_{1}\right)= & V^{*} \cup\left\{w_{1}, w_{2}\right\}, \\
A\left(D_{1}\right)= & \left(\bigcup_{i=2}^{q_{3}}\left\{\left(x,\left(q_{1}, 1, i-1\right)\right) \mid x \in S_{2}\left(\left(q_{1}, i\right)\right)\right\}\right) \\
& \cup\left(\bigcup_{i=2}^{q_{2}}\left\{\left(x,\left(q_{1}, i-1, q\right)\right) \mid x \in S_{3}\left(\left(q_{1}, i\right)\right)\right\}\right) \\
& \cup\left\{\left(x, w_{1}\right) \mid x \in S_{2}\left(\left(q_{1}, 1\right)\right)\right\} \cup\left\{\left(x, w_{2}\right) \mid x \in S_{3}\left(\left(q_{1}, 1\right)\right)\right\} .
\end{aligned}
$$

The ordering obtained by adding $w_{1}, w_{2}$ on the head of the lexicographic ordering of $V^{*}$ is an acyclic ordering of $D_{1}$, and let $w_{1}, w_{2}, \ldots, w_{q_{2} q_{3}+2}$ be the ordering. In addition,

$$
\mathcal{N}\left(D_{1}\right)=\left\{S_{2}\left(\left(q_{1}, i\right)\right) \mid i \in\left[q_{3}\right]\right\} \cup\left\{S_{3}\left(\left(q_{1}, i^{\prime}\right)\right) \mid i^{\prime} \in\left[q_{2}\right]\right\} .
$$

Therefore $D_{1}$ is an acyclic digraph such that $C\left(D_{1}\right)=H^{*} \cup\left\{w_{1}, w_{2}\right\}$.

Note that, for $\left(p_{2}, p_{3}\right) \in\left[q_{2}\right] \times\left[q_{3}\right]$, the clique in $K_{q_{1}} \square K_{q_{2}} \square K_{q_{3}}$ obtained by deleting the vertex $\left(q_{1}, p_{2}, p_{3}\right)$ from an element $S_{1}\left(\left(p_{2}, p_{3}\right)\right)$ of $K_{q_{1}} \square K_{q_{2}} \square K_{q_{3}}$ 
is a maximal clique of $K_{q_{1}-1} \square K_{q_{2}} \square K_{q_{3}}$. Then by (10), for each $\left(p_{2}, p_{3}\right) \in$ $\left[q_{2}\right] \times\left[q_{3}\right]$, the set $\left\{v \in V\left(D_{0}\right) \mid N_{D_{0}}^{-}(v)=S_{1}\left(\left(p_{2}, p_{3}\right)\right) \backslash\left\{\left(q_{1}, p_{2}, p_{3}\right)\right\}\right\}$ is not empty, and so we take an element $y_{\left(p_{2}, p_{3}\right)}$ of this set.

Now we define a digraph $D$ as follows:

$$
\begin{aligned}
& V(D)=V\left(K_{q_{1}} \square K_{q_{2}} \square K_{q_{3}}\right) \cup I_{6}, \\
& A(D)=A\left(D_{0}\right) \cup A\left(D_{1}\right) \cup\left\{\left(\left(q_{1}, p_{2}, p_{3}\right), y_{\left(p_{2}, p_{3}\right)}\right) \mid\left(p_{2}, p_{3}\right) \in\left[q_{2}\right] \times\left[q_{3}\right]\right\} .
\end{aligned}
$$

Note that since $N_{D_{0}}^{-}\left(w_{1}\right)=N_{D_{0}}^{-}\left(w_{2}\right)=\emptyset$,

$$
\left\{N_{D}^{-}\left(w_{1}\right), N_{D}^{-}\left(w_{2}\right)\right\}=\left\{S_{2}\left(\left(q_{1}, 1\right)\right), S_{3}\left(\left(q_{1}, 1\right)\right)\right\} .
$$

By the definition of $D$ and (10), (11), and (12), we can conclude that $\mathcal{N}(D)=$ $\mathcal{F}_{\left(q_{1}, q_{2}, q_{3}\right)}$ and so $E(C(D))=E\left(K_{q_{1}} \square K_{q_{2}} \square K_{q_{3}}\right)$. Thus,

$$
C(D)=\left(K_{q_{1}} \square K_{q_{2}} \square K_{q_{3}}\right) \cup I_{6} .
$$

Then the ordering

$$
v_{1}, v_{2}, \ldots, v_{q_{1} q_{2} q_{3}-q_{2} q_{3}+6}, w_{3}, w_{4}, \ldots, w_{q_{2} q_{3}+2}
$$

of the vertices of $D$ is an acyclic ordering. To see this, take an arc $a=(x, y) \in$ $A(D)$. If $a \in A\left(D_{0}\right) \cup A\left(D_{1}\right)$, then $y$ appears before $x$ in (13), since $D_{0}$ and $D_{1}$ are acyclic. If $a \notin A\left(D_{0}\right) \cup A\left(D_{1}\right)$, then $x \in\left\{w_{3}, w_{4}, \ldots, w_{q_{2} q_{3}+2}\right\}$ and $y \in\left\{v_{1}, v_{2}, \ldots, v_{q_{1} q_{2} q_{3}-q_{2} q_{3}+6}\right\}$, thus $y$ appears before $x$ in (13). Thus the digraph $D$ is acyclic. Hence the lemma holds.

Proof of Theorem 5. By Lemma 14, we have $k(H(3, q)) \geq 6$ for $q \geq 3$. By Lemma 15 , we have $k(H(3, q))=k\left(K_{q} \square K_{q} \square K_{q}\right) \leq 6$ for $q \geq 3$. Hence Theorem 5 holds.

\section{Concluding remarks}

In this paper, we gave the exact values of the competition numbers of Hamming graphs with diameter 2 or 3.

We conclude this paper with leaving the following questions for further study:

- What is the competition number of a Hamming graph $H(4, q)$ with diameter 4 for $q \geq 3$ ?

- Give the exact values or a good bound for the competition numbers of Hamming graphs $H(n, q)$.

\section{References}

[1] H. H. Cho and S.-R. Kim, The competition number of a graph having exactly one hole, Discrete Math. 303 (2005), no. 1-3, 32-41.

[2] J. E. Cohen, Interval Graphs and Food Webs: a finding and a problem, Document 17696-PR, RAND Corporation, Santa Monica, CA, 1968.

[3] _ Food Webs and Niche Space, Princeton University Press, Princeton, NJ, 1978.

[4] S.-R. Kim, The competition number and its variants, Quo vadis, graph theory?, 313-326, Ann. Discrete Math., 55, North-Holland, Amsterdam, 1993. 
[5] - On competition graphs and competition numbers, Commun. Korean Math. Soc. 16 (2001), no. 1, 1-24.

[6] _ Graphs with one hole and competition number one, J. Korean Math. Soc. 42 (2005), no. 6, 1251-1264.

[7] S.-R. Kim, J. Y. Lee, and Y. Sano, The competition number of a graph whose holes do not overlap much, Discrete Appl. Math. 158 (2010), no. 13, 1456-1460.

[8] S.-R. Kim, B. Park, and Y. Sano, The competition numbers of Johnson graphs, Discuss. Math. Graph Theory 30 (2010), 449-459.

[9] S.-R. Kim and F. S. Roberts, Competition numbers of graphs with a small number of triangles, Discrete Appl. Math. 78 (1997), no. 1-3, 153-162.

[10] S.-R. Kim and Y. Sano, The competition numbers of complete tripartite graphs, Discrete Appl. Math. 156 (2008), no. 18, 3522-3524.

[11] J. Y. Lee, S.-R. Kim, S.-J. Kim, and Y. Sano, The competition number of a graph with exactly two holes, Ars Combin. 95 (2010), 45-54.

[12] R. J. Opsut, On the computation of the competition number of a graph, SIAM J. Algebraic Discrete Methods 3 (1982), no. 4, 420-428.

[13] B. Park, S.-R. Kim, and Y. Sano, On competition numbers of complete multipartite graphs with partite sets of equal size, preprint RIMS-1644 October 2008. (http://www.kurims.kyoto-u.ac.jp/preprint/file/RIMS1644.pdf)

[14] _ The competition numbers of complete multipartite graphs and mutually orthogonal Latin squares, Discrete Math. 309 (2009), no. 23-24, 6464-6469.

[15] B. Park and Y. Sano, The competition numbers of ternary Hamming graphs, Appl. Math. Lett. 24 (2011), 1608-1613.

[16] F. S. Roberts, Food webs, competition graphs, and the boxicity of ecological phase space, Theory and applications of graphs (Proc. Internat. Conf., Western Mich. Univ., Kalamazoo, Mich., 1976), pp. 477-490. Lecture Notes in Math., Vol. 642, Springer, Berlin, 1978.

[17] Y. Sano, The competition numbers of regular polyhedra, Congr. Numer. 198 (2009), 211-219.

[18] Y. Wu and J. Lu, Dimension-2 poset competition numbers and dimension-2 poset double competition numbers, Discrete Appl. Math. 158 (2010), no. 6, 706-717.

BORAM PARK

Department of Mathematics Education

Seoul National University

SeOul 151-742, Korea

E-mail address: kawa22@snu.ac.kr

YOSHIO SANO

NATIONAL Institute OF INFORMATICS

TOKYO 101-8430, JAPAN

E-mail address: sano@nii.ac.jp 